\title{
A Novel Array-Type Transcranial Direct Current Stimulation (tDCS) System for Accurate Focusing on Targeted Brain Areas
}

\author{
Ji-Hye Park ${ }^{1,2}$, Seung Bong Hong ${ }^{3}$, Do-Won Kim ${ }^{1,2}$, Minah Suh $^{4}$, and Chang-Hwan $\operatorname{Im}^{1}$ \\ ${ }^{1}$ Department of Biomedical Engineering, Hanyang University, Seoul 133-791, Korea \\ ${ }^{2}$ Department of Biomedical Engineering, Yonsei University, Wonju 220-710, Korea \\ ${ }^{3}$ Department of Neurology, Samsung Medical Center, School of Medicine, Sungkyunkwan University, Seoul 110-745, Korea \\ ${ }^{4}$ Department of Biological Science, Sungkyunkwan University, Suwon 440-746, Korea
}

In this paper, we propose a new array-type transcranial direct current stimulation (tDCS) system, which can modulate cortical excitability of human brain in a more effective manner. Once a target location inside a brain is determined, optimal injection current/potential at each electrode is calculated automatically by solving a constrained optimization problem. Current density distribution in a realistic head model was evaluated using the 3-D finite element method (FEM) adopting the superposition principle. Simulation results demonstrated that the proposed tDCS system enables effective and accurate field concentration on targeted brain areas.

Index Terms-Electromagnetic brain stimulation, finite element method (FEM), transcranial direct current stimulation (tDCS).

\section{INTRODUCTION}

$\mathbf{T}$ RANSCRANIAL direct current stimulation (tDCS) is a kind of noninvasive brain electrical stimulation technique, which can modulate excitability of the cerebral cortex with a small direct current transmitted from a pair of scalp electrodes [1]. tDCS has been applied to a variety of clinical applications, especially to the treatment of various neuropsychiatric diseases and neurological disorders such as depression, epilepsy, electroanalgesia, stroke, and Parkinson's disease [1]-[5]. Although the effect of tDCS has turned out to be similar to that of repetitive transcranial magnetic stimulation (rTMS), tDCS has been attracting great attention from the neuroscience society since it has several advantages over the traditional rTMS treatments [6], [7]. Compared to rTMS, tDCS stimulates relatively wider brain regions, but is thought to be appropriate for the stimulation of relatively deeper brain areas than rTMS because the magnetic induction current elicited by rTMS is generally distributed at shallow cortical areas [7]. Moreover, tDCS is specifically useful for patients who need long-term or frequent therapies because the patients cannot move their heads at all during rTMS therapy, whereas they can freely move their heads during tDCS therapy. The tDCS systems can be used even under mobile environments if they are installed in a wheelchair or a bed. Lastly, tDCS can be applied to some patients with metallic implants or a heart pacemaker in their body, for whom rTMS cannot be used [6], [8].

Conventional tDCS systems have generally used two scalp electrodes called anode $(+)$ and cathode $(-)$, whose locations have been usually determined empirically considering the location of a targeted brain area. However, a series of computer simulation studies have demonstrated that such empirical determination of electrode locations might lead to inaccurate targeting due to the edge effect and inhomogeneous conductivity distribution inside the human head [9], [10], that is to say the intrinsic

Manuscript received May 31, 2010; accepted August 23, 2010. Date of current version April 22, 2011. Corresponding author: C.-H. Im (e-mail: ich@hanyang.ac.kr).

Color versions of one or more of the figures in this paper are available online at http://ieeexplore.ieee.org.

Digital Object Identifier 10.1109/TMAG.2010.2072987 characteristics of conductive currents that tend to flow along the shortest path with smallest resistivity. Recently, Im et al. [10] attempted to optimize the scalp electrode locations for a given target brain area by applying 3-D finite element method (FEM) and the $(2+6)$ evolution strategy (ES) algorithm. Although their approach provided us with a new way for individualized brain stimulation, it was hard to be applied directly to clinical applications due to the problem complexity and heavy computational burden. Abhishek et al. [8] also tested various electrode configurations to find out one resulting in the most focused electric field distributions. Although their simulation results demonstrated that a ring-type electrode configuration could yield better field concentration, the configuration was effective only at shallow brain areas and thus could hardly be used for general purposes.

In this paper, we propose a new tDCS system that uses arraytype multiple electrodes. Instead of adjusting the electrode locations, the proposed system fixes the locations of two electrode arrays and determines their optimal injection current distributions by solving a constrained optimization problem. Considering that the given electric field analysis problem is linear, the superposition principle is adopted to facilitate more efficient computation of objective functions.

\section{Methods AND MATERIALS}

\section{A. Head Modeling and FEM}

Analysis of current conduction is essential in the design of electrical stimulation systems. Conventional studies have used a simplified head model [2] or a single-slice brain model [12] for the numerical analysis of 3-D conduction current inside a human head. In this letter, a full 3-D analysis was performed using a 3-D FEM solver [10]. Fig. 1 shows the head model used for the present simulation study, which consists of scalp, skull, and cerebrospinal fluid (CSF) regions. Since the inhomogeneous electrical conductivity distribution of a human head cannot be estimated accurately even with the currently best imaging modalities, we used widely accepted effective electrical conductivity values of head structures. The effective electrical conductivity values for the scalp, skull, and CSF/brain regions were set to well-known constants 0.22 , 0.014 , and $1.79(\mathrm{~S} / \mathrm{m})$, respectively [13]. The finite element 


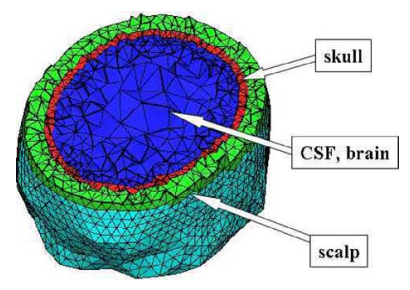

Fig. 1. Finite element head model used for the present simulation study. The effective conductivity values for scalp, skull, and CSF/brain regions were set as $0.22,0.014$, and $1.79(\mathrm{~S} / \mathrm{m})$, respectively [10], [13].

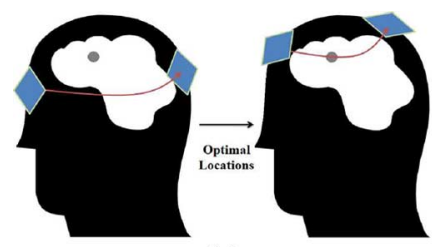

(a)

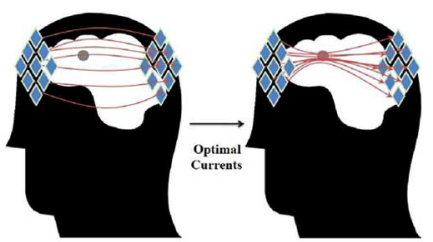

(b)
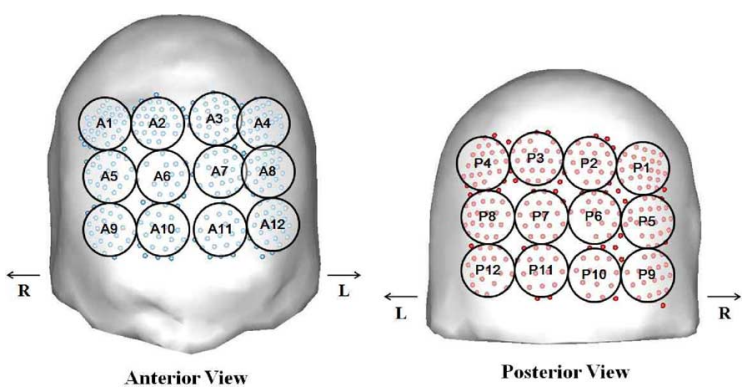

Fig. 3. Array-type electrode configurations used for the test simulations: (left) anterior view and (right) posterior view. "R" and "L" represent "right" and "left," respectively. "A" and "P" in the electrode names represent "anterior" and "posterior," respectively. Two electrodes located at opposite (symmetric) positions (electrodes with an identical number, e.g., A7 and P7) have the same current amplitudes, but have opposite signs (e.g., A7: +0.3 mA, P7: $-0.3 \mathrm{~mA}$ ).

e.g., A7 and P7 in Fig. 3) were assumed to have an identical current amplitude, but have opposite signs (e.g., A7: $+0.3 \mathrm{~mA}$; P7: $-0.3 \mathrm{~mA}$ ). Before the optimization process, we first stored 12 FEM results, each of which was obtained applying a unit input value between each pair of electrodes (Dirichlet boundary conditions were imposed at nodes in each electrode area). Since the present electrostatic problem is mathematically linear, the superposition principle could be applied. When the input values at 12 pairs of electrodes were given, the electric potentials at any nodes could be evaluated by linearly combining the precalculated FEM results with the input value at each electrode pair. We confirmed that the errors between the potential values obtained from the superposition principle and the normal FEM computation were negligible (error-to-potential ratio $<0.01$ ).

\section{Determination of Optimal Injection Currents}

To determine the optimal injection currents, we solved a constrained optimization problem. The optimization variables were input electrical potentials at electrodes in an anode array.

Once the input values of the anode array were given, those in a cathode array could be determined automatically. An objective function to be minimized and constraints were defined as follows:

$$
\begin{array}{ll}
\text { Maximize } & f=\sum_{i=1}^{M}\left|J_{i}\right| \\
\text { subject to } & \sum_{n=1}^{N} x_{n}=\text { const } \cdot \text { and } x_{n} \geq 0
\end{array}
$$

where $x_{n}$ represents the input potential value at the $n$th electrode, $N$ is the number of electrodes in an array $(N=12$ herein), $J_{i}$ is the current density evaluated at the $i$ th node included in a target brain area, and $M$ represents the number of nodes belonging to the target brain area. We first evaluated the current density values of elements, and then transformed them into nodal values. The total amount of the potentials was restricted to be a constant value, which was set to 1 in the simulation, to prevent the values from diverging to infinity. After the optimization, optimal injection currents could also be obtained by integrating the current density at each electrode.

Among various algorithms to solve the above constrained optimization problem, we selected an algorithm based on the Nelder-Mead simplex method [15] since the derivative of circle with a $2-\mathrm{cm}$ radius (Fig. 3). Two electrodes located at opposite positions (electrodes with an identical number, 


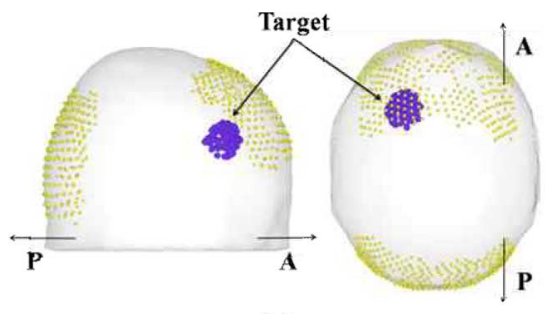

(a)

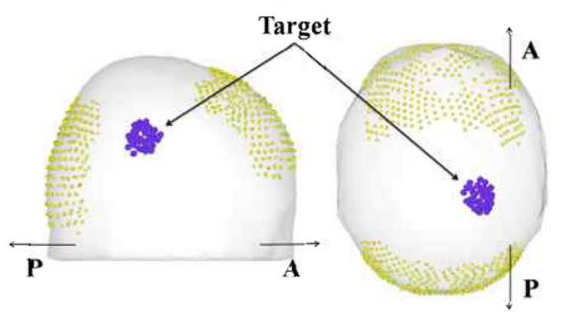

(b)

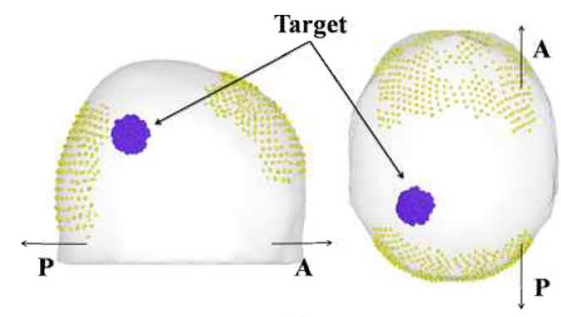

(c)

Fig. 4. Locations of three target brain areas: (a) left frontal area, (b) right inferior parietal area (deeper than the others), and (c) left superior parietal area. "A" and "P" represent "anterior" and "posterior," respectively.

the objective function could not be expressed as an analytic form. We used a slightly modified version of a MATLAB (The MathWorks, Natick, MA, ver. 7.7.0) code called SIMPS (Strategy Simplex; http://www.mathworks.com/matlabcentral/ fileexchange/102-sim), where the constraints were considered by penalizing the target function. The algorithm was based on the typical nonlinear simplex implementation [15], but improved its performance by adopting a local minimizer. The iteration was stopped when there was no further improvement in the penalized objective function value. Since we applied the superposition principle, the time taken to obtain the optimal current distribution did not exceed $5 \mathrm{~min}$, whenever the optimization was performed under a Pentium IV 3.2-GHz personal computer environment (computation of the 12 basis FEM results took about $2 \mathrm{~min}$ ). Please note that it took about $2 \mathrm{~h}$ to optimize the electrode locations under the same computer environment according to our previous report [10].

\section{Simulation AND Results}

For the simulation model presented in Fig. 1, we selected three different target brain areas: left frontal area, right inferior parietal area, and left superior parietal area. The target areas were arbitrarily selected to test various locations and depths. Fig. 4 shows the three target brain areas. In our simulations, electrode arrays in the posterior region were assumed to be the anode electrodes. The initial values of the optimization were identically set to $0.083(=1 / 12)$. Since the Nelder-Mead simplex method is a kind of deterministic optimization algorithms, the results always converged to a single optimum.

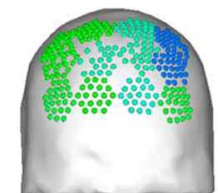

Anterior View

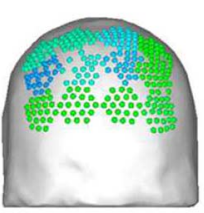

Anterior View

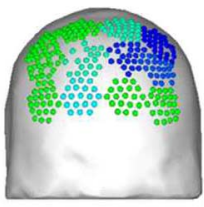

Anterior View

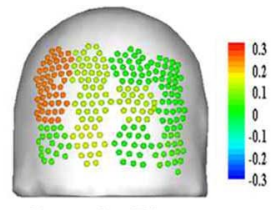

Posterior View

(a)

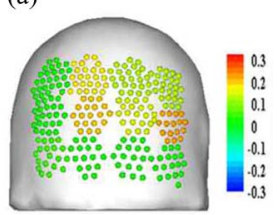

Posterior View

(b)

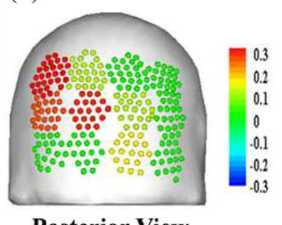

(c)
Fig. 5. Optimized current distributions. Current distribution was illustrated instead of potential distribution as most tDCS systems use constant current sources. Colors represent the injection currents (mA). The target brain regions are (a) left frontal, (b) right inferior parietal, and (c) left superior parietal areas.

TABLE I

Averaged CurRent Density at TARget Areas Evaluated Before AND AFTER THE OPTIMIZATION PROCESS $\left(\mathrm{mA} / \mathrm{m}^{2}\right)$

\begin{tabular}{ccc}
\hline Target Locations & Before Optimization & After Optimization \\
\hline Left Frontal & 11.117 & 15.030 \\
Right Inferior Parietal & 4.739 & 6.716 \\
Left superior Parietal & 26.707 & 47.589 \\
\hline
\end{tabular}

Fig. 5 shows the optimal input currents determined for the three target areas, where the current values were normalized so that the total current equals $1 \mathrm{~mA}$, which has been regarded as a reasonable current level [3], [4], [8]. It can be seen from the figures that the amplitude of injection currents near the target areas have relatively higher amplitudes, from which we could indirectly confirm the success of the optimization. The results also suggest that optimizing the injection current distribution has a similar effect to physically adjusting electrode locations.

Fig. 6 shows the directions and magnitudes of the current density vectors before and after the optimization, where the streamlines show the current flow starting from various locations in the anode array. The left three columns show the current flows obtained for the initial condition, while right three columns show those for the optimal currents. Table I shows the current density values at three target areas evaluated before and after the optimization. It can be seen that the average current density values at the target areas were increased after the optimization, with rates of increase ranging from $35 \%$ to $78 \%$, which implies that less current injection is necessary to obtain equivalent stimulation effects. We also evaluated average current density at 30 randomly selected nontarget regions and observed $11.8 \%$ (left frontal), $14.2 \%$ (right inferior parietal), and $7.3 \%$ (left superior parietal) reduction of current density after the optimization. 

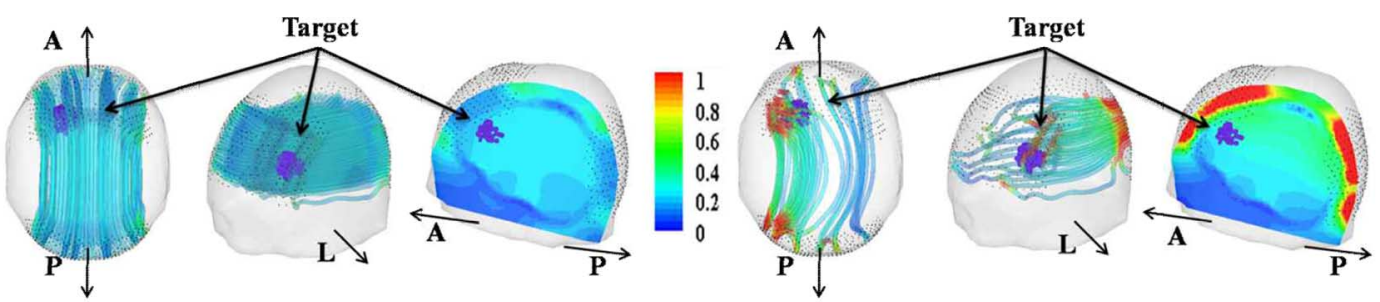

(a)
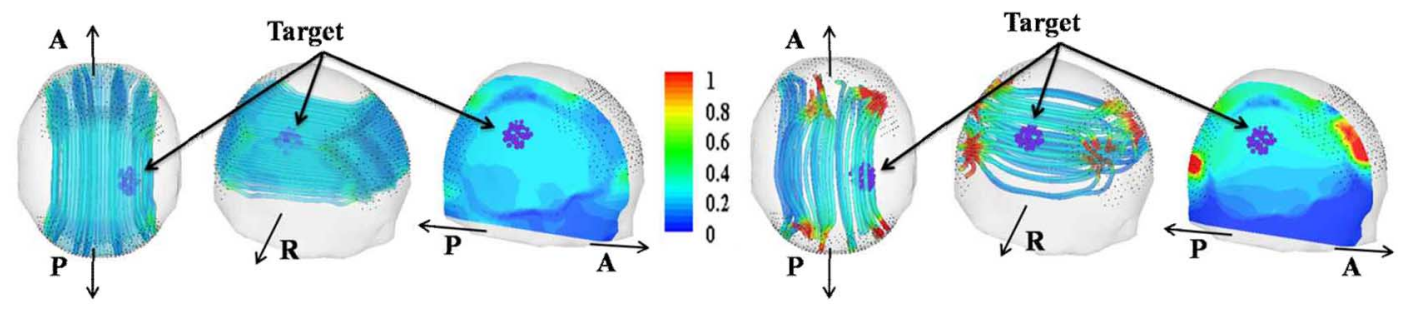

(b)
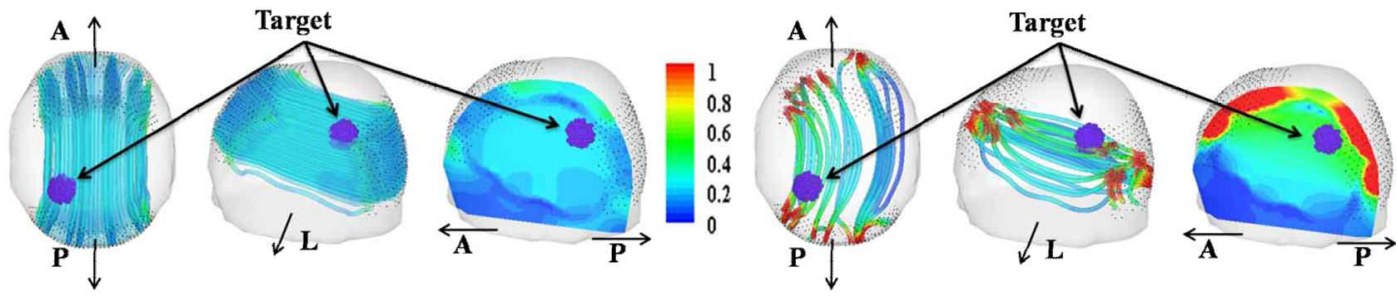

(c)

Fig. 6. Directions and magnitudes of the current density vectors before and after the optimization. The target locations of (a)-(c) correspond to those of (a)-(c) in Fig. 4. The left three figures in each of the simulation results show the current flows obtained for the initial value (before the optimization), while the right three figures show those for the optimal currents (after the optimization). The streamlines show the current flow starting from various locations in the anode electrode array, and the colors represent the magnitude of the current density (normalized with respect to maximum; A: anterior; P: posterior; $\mathrm{R}$ : right; L: left).

\section{CONCLUSION}

In this paper, we proposed a new array-type tDCS system, which is capable of shaping current flow around targeted brain areas by controlling the input values of the array electrodes. Based on this study, we are currently implementing an MRIguided individualized brain dc stimulation system, which can consider individual differences in brain anatomy as well as the locations of brain lesions.

\section{ACKNOWLEDGMENT}

This work was supported in part by the National Research Foundation of Korea (NRF) under Grant 2010-0007015 funded by the Korean Government and by the Korea Healthcare Technology R\&D Project, Ministry for Health, Welfare \& Family Affairs, Republic of Korea, under Grant A090579-0901-0000201.

\section{REFERENCES}

[1] B. Gregory, T. Trung, M. Steven, and B. Paul, "The use of tDCS and CVS as methods of non-invasive brain stimulation," Brain Res. Rev., vol. 56, pp. 346-361, 2007.

[2] P. C. Miranda, M. Lomarev, and M. Hallett, "Modeling the current distribution during transcranial direct current stimulation," Clin. Neurophysiol., vol. 117, pp. 1623-1629, 2006.

[3] F. Fregni, P. S. Boggio, M. Nitsche, and A. Pascual-Leone, "Transcranial direct current stimulation," Br. J. Psychiatry, vol. 186, pp. 446-447, 2005.

[4] F. Fregni and A. Pascual-Leone, "Technology insight: Noninvasive brain stimulation in neurology-perspectives on the therapeutic potential of rTMS and tDCS," Nat. Clin. Pract. Neurol., vol. 3, pp. 383-393, 2007.
[5] F. Fregni, S. Thome-Souza, M. A. Nitsche, S. D. Freedman, K. D. Valente, and A. Pascual-Leone, "A controlled clinical trial of cathodal DC polarization in patients with refractory epilepsy," Epilepsia, vol. 47, pp. 335-342, 2006.

[6] A. Priori, M. Hallett, and J. C. Rothwell, "Repetitive transcranial magnetic stimulation or transcranial direct current stimulation?," Brain Res., vol. 2, pp. 241-245, 2009.

[7] C.-H. Im and C. Lee, "Computer-aided performance evaluation of a multi-channel transcranial magnetic stimulation system," IEEE Trans. Magn., vol. 42, no. 12, pp. 3803-3808, Dec. 2006.

[8] R. Sparing and F. M. Mottaghy, "Noninvasive brain stimulation with transcranial magnetic or direct current stimulation (TMS/tDCS)-from insights into human memory to therapy of its dysfunction," Methods, vol. 44, pp. 329-337, Apr. 2008.

[9] T. Wagner, F. Fregni, S. Fecteau, A. Grodzinsky, M. Zahn, and A. Pascual-Leone, "Transcranial direct current stimulation: A computerbased human model study," NeuroImage, vol. 35, pp. 1113-1124, Apr. 2007.

[10] C. H. Im, H. H. Jung, J. D. Choi, S. Y. Lee, and K. Y. Jung, "Determination of optimal electrode positions for transcranial direct current stimulation (tDCS)," Phys. Med. Biol., vol. 53, pp. N219-N225, May 2008.

[11] D. Abhishek, E. Maged, B. Fortunato, and M. Bikson, "Transcranial current stimulation focality using disc and ring electrode configurations: FEM analysis," J. Neural Eng., vol. 5, pp. 163-174, 2008.

[12] R. N. Holdefer, R. Sadleir, and M. J. Russell, "Predicted current densities in the brain during transcranial electrical stimulation," Clin. Neurophysiol., vol. 117, pp. 1388-1397, 2006.

[13] J. Haueisen, C. Ramon, M. Eiselt, H. Brauer, and H. Nowak, "Influence of tissue resistivities on neuromagnetic fields and electric potentials studied with a finite element model of the head," IEEE Trans. Biomed. Eng., vol. 44, no. 8, pp. 727-735, Aug. 1997.

[14] J. Jin, The Finite Element Method in Electromagnetics, 2nd ed. New York: Wiley, 2002.

[15] J. C. Lagarias, J. A. Reeds, M. H. Wright, and P. E. Wright, "Convergence properties of the nelder-mead simplex method in low dimensions," SIAM J. Optim., vol. 9, pp. 112-147, 1998. 